\title{
Optimal Placement of Capacitor in RDS Using Modified Cuckoo Search Algorithm
}

\author{
M.S.Giridhar ${ }^{1}$, S.Sivanagaraju ${ }^{2}$ \\ Associate Professor, Department of EEE, SITAMS, Chittoor, India ${ }^{1}$ \\ Professor, Department of EEE, J.N.T.U College of Engineering, Kakinada, India ${ }^{2}$
}

\begin{abstract}
A modified cuckoo Search technique is used for Capacitor Placement in Radial Distribution System with the Objectives of maximization of savings, minimization of voltage deviation and minimization of section current injection are used independently to find the Optimal Size of capacitors. The Power Loss Indices are used for location of Capacitor in System. A Novel procedure is used for location of capacitors by taking the Total Active Power loss minimum of the system as objective. To demonstrate the performance of the developed algorithm, a standard 15-node system is considered. The results obtained with the proposed method are better when compared with the existing method.
\end{abstract}

Keywords: Modified Cuckoo Search Algorithm, Optimal Capacitor Placement, Savings, Voltage deviation.

\section{INTRODUCTION}

With the usage of different types of electronic loads the customer loads (domestic) loads draw huge amount of reactive currents due to which there is a drop in voltage due to quadrature component of voltage, also the system line resistive losses increases, so, there is a need to compensate the reactive current by placing appropriate amount (kVAr) of capacitor at particular nodes so that the reactive power is compensated. The installation of shunt capacitors in the distribution feeder reduces the peak power and energy losses by improving the voltage profile. The objectives for capacitor placement is to minimize the total real power loss in a given distribution system as well as to maximize the savings in annual energy loss in the system with installation of capacitors. The government authorities are interested in the investment cost of capacitors as well as the environmental effects, the operator is concerned about the number of capacitors and size of the capacitors to be placed at particular nodes. The system planner is concerned about the economic benefits that can be obtained such as the annual energy loss minimization as well as he benefit due to voltage profile improvement. So, the capacitor placement problem is an multi-objective optimization problem. Many authors have used the single objective function for optimal placement of capacitors, with each objective function given weight values, this is one way of dealing the multi-objective optimization problem. Resent progress in multi-objective optimization problems is the usage of Evolutionary methods such as multi-objective genetic algorithms, nondominant Pareto search algorithm, e-constraint method. To identify the candidate location and the capacitor placement problem was solved using LSF [5] and the optimal capacitor size is determined through Plant Growth Simulation Algorithm (PGSO). An Extended Dynamic Programming (EDP) method capable of providing a global optimal solution with pseudo-polynomial complexity in worst case and with linear complexity for practical applications has been proposed in [1]. Employment of Particle Swarm Optimization (PSO) for identifying locations, type and size of capacitor to be placed taking into account of Harmonic distortion effect, discrete nature of capacitors and different load levels [2]. Kalyuzhny.A et al., [3] approached a solution for placing shunt capacitors which is found to be good for system of feeders fed through their transformers and not for any individual feeders. Genetic algorithm is used to optimize the sizing of capacitor which is now implemented in Isreal Electric Corporation (IECo). Ji-Pyng Chiou et al., [4] identified an effective method namely Variable Scaling Hybrid Differential Evolution (VSHDE) where the drawback of Hybrid Differential Evolution (HDE) is suppressed by variable scaling factor in VSHDE. S.M. Tabatabaei and B. Vahidi [6] proposed a fresh method for placing shunt capacitors in a distribution system where the node for installation is identified by fuzzy reasoning based on fuzzy set theory. The determination of optimal node for capacitor is achieved by Bacterial Foraging Algorithm (BFA). This method results as an economic solution for reducing energy loss, power loss and total capacitive compensation.

\section{MATHEMATICAL FORMULATIONS}

The proposed method is developed based on a derived matrix node-injection to branch current matrix and equivalent current injections. In this section, the developed procedure will be described in detail.

For distribution networks, the equivalent current injection based model is more practical [3]. For node-i, the complex load $S_{\mathrm{I}}$ is expressed as

$$
S_{i}=P_{i}+j \times Q_{i} \quad \mathrm{i}=1,2 \ldots . \text { nnode }
$$

and the corresponding equivalent current injection at the $\mathrm{k}^{\text {th }}$ iteration of solution is

$$
I_{i}^{k}=\left[\frac{P_{i}+j \times Q_{i}}{V_{i}^{k}}\right]^{*}
$$


Where $\mathrm{V}_{\mathrm{i}}^{\mathrm{k}}$ and $\mathrm{I}_{\mathrm{i}}^{\mathrm{k}}$ are the node voltage and equivalent current injection of node-i at the $\mathrm{k}^{\text {th }}$ iteration, respectively.

$$
B_{j}=\sum_{i=2}^{\text {nnode }} I_{i} \forall j=1,2, \ldots n b r
$$

The receiving end node voltages are found by a forward sweep through the ladder network using the generalized equation as

$$
V_{m 2}=V_{m 1}-B_{j} Z_{j}
$$

Where, $\mathrm{m} 2$ and $\mathrm{m} 1$ are the variables for receiving end and sending end node as

The real and reactive power loss of branch $\mathrm{j}$ is given by [9].

$$
\begin{aligned}
& P_{\text {loss }}^{j}=B_{j}^{2} \times R_{j} \text { for } \mathrm{j}=1,2, \ldots \text { nbr. } \\
& Q_{\text {loss }}^{j}=B_{j}^{2} \times X_{j} \text { for } \mathrm{j}=1,2, \ldots . \mathrm{nbr}
\end{aligned}
$$

\section{III.OPTIMIZATION PROBLEM FORMULATION WITH CAPACITOR}

To formulate optimization problem with capacitor, in this chapter, three objectives namely, savings, voltage deviation (Vdev) and section current index (SCI) are considered. The details are given as follows:

\section{A. Maximization of Savings (Rs)}

This objective is used to maximize the savings in a given system in the presence of capacitor. The mathematical expression used to calculate the savings is given as follows:

$$
\text { Savings }=\max [\mathrm{KP}+\mathrm{KF}+\mathrm{KE}-\mathrm{KC}]
$$

Where, $\mathrm{S}$ is the net savings in Rupees.

$\mathrm{KP}$ is the benefit in Rupees due to reduced demand in $\mathrm{kW}$ $\mathrm{KF}$ is the benefit in Rupees due to released feeder capacity in $\mathrm{kVA}$

$\mathrm{KE}$ is the benefit in Rupees due to saving in energy in $\mathrm{kWh}$

$\mathrm{KC}$ is Cost of installation of the capacitor in Rupees

The individual terms can be expressed in detailed as follows:

\section{(a) Benefit due to reduced demand}

$$
\mathrm{KP}=\Delta \mathrm{KP} \times \mathrm{CKP} \times \mathrm{IKP}
$$

Here, $\triangle \mathrm{KP}$ is the reduced demand $(\mathrm{kW})$

CKP is the cost of generation ( taken as Rs.10,000/kW)

IKP is the depreciation cost for generation (taken as 0.2)

\section{(b) Benefit due to released feeder capacity}

$$
\mathrm{KF}=\Delta \mathrm{KF} \times \mathrm{CKF} \times \mathrm{IKF}
$$

Here, $\triangle \mathrm{KF}$ is the released feeder capacity (KVA) $\mathrm{CKF}$ is the cost of the feeder (taken as Rs.171.5/KVA) IKF is depreciation cost of the feeder (taken as 0.2)

\section{(c) Benefit due to savings in energy}

$$
\mathrm{KE}=\Delta \mathrm{KF} \times \mathrm{R}
$$

Here, $\Delta \mathrm{KF}$ is the savings in energy $=$ (annual energy losses before installing the capacitor - annual energy losses after installing capacitor) (KWh) $\mathrm{R}$ is the cost of energy (taken as Rs. 6/kWh)

\section{(d) Cost of installation of Capacitor}

$$
\mathrm{KC}=\mathrm{Q}_{\mathrm{c}} \times \mathrm{ICKC} \times \mathrm{IKC}
$$

Here, $Q_{c}$ is the total $\mathrm{kVAr}$

ICKC is the cost of the capacitor (Rs. 200/kVAr)

IKC is the depreciation cost of the capacitor (taken as 0.2)

\section{A. Minimization of Voltage deviation $\left(V_{d e v}\right)$ (p.u.)}

It is necessary to main the voltage magnitude at the nodes within permissible limits to increase the security of the system. For this, it is necessary to minimize the voltage deviation at system nodes. The system voltage deviation can be calculated as

$$
\left.\mathrm{V}_{\mathrm{dev}}=\min \left(\sum_{j=2}^{\text {nnode }}\left(\left|V_{j}\right|-\left|V_{\text {rated }}\right|\right)^{2}\right)\right)(p . u)
$$

Where, $\mathrm{Vj}$ is the voltage magnitude at $\mathrm{jth}$ node and Vrated is the rated voltage considered to be 1.0 p.u. and 'nnode' is the total number of nodes in the system.

\section{B. Minimization of Section current index (SCI)}

Providing the active and reactive power near the loads may increase or decrease the current flow in some sections of the network, thus releasing more capacity or also place out of distribution line limits. The section current index (SCI) gives important information about the level of currents through the network. The section current index can be calculated when performing the power flow analysis before and after installation of capacitor banks as

$$
\mathrm{SCI}=\left[\min \left(\frac{\sum_{s=1}^{L} \frac{I_{s m}-I_{s a s}}{\max \left(I_{s m}, I_{s a s}\right)}}{L}\right)\right]
$$

Where

$I_{s m}$ is the mean of Line section current after placement of capacitor.

$I_{\text {sas }}$ is the line section current after placement of Capacitor

L total number of line section

$\mathrm{S}$ is the line section

\section{Constraints}

The following equality and inequality considered for the optimization problem

Equality Constraints:

$$
Q_{s / s}=\sum_{j=2}^{n b u s} Q_{\text {load }}^{j}+\sum_{k=1}^{n b r} Q_{\text {loss }}^{k}-\sum_{h=1}^{n d g} Q_{C}^{h}
$$

Where ' $j$ ' superscript stands for node number, ' $k$ ' for branch number and ' $h$ ' is for Capacitor node number.

\section{Inequality Constraints:}

i) The node voltage magnitudes are to be kept within acceptable operating limits throughout the optimization process. That is $\pm 5 \%$ of the nominal voltage value.

$$
V_{j}^{\min } \leq V_{j} \leq V_{j}^{\max } \forall \mathrm{j}=2 \ldots \text { nnode }
$$


ii) Capacitor-unit size

$$
0 \leq Q_{C}^{j} \leq Q_{C}^{\max } \forall \mathrm{j}=2 \ldots \text { nnode }
$$

\section{Optimal location of capacitor}

The proposed location of capacitor is explained with a 15 node radial distribution system [7]. The Total active power losses of the system are calculated by running the load flows for base load, which is obtained as $59.1096 \mathrm{~kW}$. By compensating the reactive power at each node by their respective reactive loads at that nodes one at a time, except the source node, the total active power losses in each case are computed, so, we will get (14 total active power losses for 15 node system). The power loss index (PLI) are calculated as [11]

$$
P L I(k)=\frac{L R(k)-\min (L R)}{(\max (R)-\min (R))}
$$

The best location of node for capacitor placement is identified by the PLI values obtained, i.e the nodes with PLI values greater than 0.6 are arranged in descending order and the first three nodes are identified as the capacitor locations. The optimal location out of these three nodes for location of capacitors is identified based on the total power losses and the system constraints.

\section{Capacitor Reactive Power Injection:}

The eq.(2.2) is modified to inject reactive power into node 'i' as

$$
\left(\mathrm{I}_{\mathrm{i}}^{\mathrm{k}}\right)^{\mathrm{Comp}}=\left(\left(\mathrm{P}_{\mathrm{i}}+\mathrm{j}^{*} \mathrm{Q}_{\mathrm{i}-\mathrm{j}} * \mathrm{Q}_{\mathrm{Comp}}\right) / \mathrm{V}_{\mathrm{i}}^{\mathrm{k}}\right)^{*} \forall \mathrm{k}=2,3,4 \ldots . \text { n node }
$$

Where $\left(\mathrm{I}_{\mathrm{i}}^{\mathrm{k}}\right)^{\text {Comp }}$ is the Load Current at node ' $\mathrm{i}$ ' after placing capacitor of $\mathrm{Q}_{\text {comp }}$ rating at node ' $\mathrm{i}$ '. For the considered 15-node system the capacitors are located at $15^{\text {th }}$ and $11^{\text {th }}$ nodes. In case of MCSA the control variable initialized as the population is the size of capacitor in kVAr.

\section{E. Overview of Existing Algorithms}

Generally, there exist several optimization techniques such as Linear Programming, Integer Programming, Quadratic Programming, Combinatorial Optimization and metaheuristic optimization methods. The classical optimization methods used in scientific applications involves hessian matrix based methods and gradient based methods. But metaheuristic algorithms are developed in solving non-differentiable and nonlinearobjective functions. The solution of problems is very difficult by using the classical optimization techniques. The metaheuristic optimization algorithms that are most widely used in scientific applications are GA, PSO, DE, ABC, CSA, GSA, HS etc.

\section{F. Modified Cuckoo Search Algorithm (MCSA)}

The cuckoo search algorithm, it is a novel technique developed for solving continuous and non linear optimization problems. This algorithm was developed from the lifestyle of cuckoo bird family [9]. The basic initiative for developing algorithm is special life style of cuckoo birds, characteristics in egg laying as well as breeding.

From the life style of cuckoo bird it is well known that cuckoo lays eggs in the host bird nest due to similarity between cuckoo and host bird eggs. Whenever cuckoo laid eggs in the host bird nest only some number of eggs will hatch up and turned into cuckoo chicks and remaining will be killed by host bird. The nest in which more number of cuckoo chicks will survive that nest will be the best nest in that area. The best habitat in any area with more number of egg survival rate gives best profit of that area.

In an optimization problem, the population can be formed as an array. In cuckoo optimization algorithm such an array is called habitat.

$$
\text { Habitat }=\left[x_{1}, x_{2}, \ldots \ldots . x_{n}\right]
$$

The profit of habitat is estimated by evaluating profit function as

$$
\text { profit }=F[\text { habitat }]=F\left[x_{1}, x_{2}, \ldots \ldots, x_{n}\right\rfloor
$$

\section{G. Proposed Modified Cuckoo Search Algorithm} (MCSA)

It is the modified version of cuckoo search optimization method. Modified cuckoo search method is developed by combining GA with actual cuckoo search process by which it is observed that such method yields to better performance. Sequential steps for Modified cuckoo search algorithm are given as follows.

\section{(a) Initialization}

Initial population of control variable is randomly generated by using,

$$
x_{a b}=x_{b}^{\min }+\operatorname{rand}(0,1) \times\left(x_{b}^{\max }-x_{b}^{\min }\right)
$$

Where, $a=1,2, \ldots \ldots, n \quad b=1,2, \ldots . ., m$

$n=$ Number of nests, $m=$ Number of control variables $x_{b}^{\min }$ and $x_{b}^{\max }$ are min. and max. limits of $b^{\text {th }}$ control variable. rand $(0,1)$ is the random number generated between $[0,1]$

\section{(b) Levy flights}

Levy flight is the search process of population of solution from the randomly generated initial population. After performing the levy flight cuckoo chooses the host nest position randomly to lay egg is given in Eqn. (21) and (23). for $i^{t h}$ cuckoo, latest solutions are generated using,

$$
x_{i}{ }^{(t+1)}=x_{i}{ }^{(t)}+s_{a b} \times \alpha \oplus \operatorname{Levy}(\lambda)
$$

Where, $\alpha$ random number between $[-1,1], \oplus$ is entry wise multiplication

$s_{a b}>0$, it is the step size, based on this only new solution is generated. step size can be calculated as

$$
\begin{aligned}
& \quad s_{a b}=x_{a b}^{t}-x_{f b}^{t} \\
& \text { Where } a, f=1,2, \ldots . ., n ; b=1,2, \ldots ., m
\end{aligned}
$$
and

$$
\operatorname{Levy}(\lambda)=\left|\frac{\Gamma(1+\lambda) \times \sin \left(\frac{\pi \times \lambda}{2}\right)}{\Gamma\left(\frac{1+\lambda}{2}\right) \times \lambda \times 2\left(\frac{\lambda-1}{2}\right)}\right|^{1 / \lambda} ; 1<\lambda \leq 3
$$


INTERNATIONAL JOURNAL OF INNOVATIVE RESEARCH IN ELECTRICAL, ELECTRONICS, INSTRUMENTATION AND CONTROL ENGINEERING

Vol. 4, Issue 2, February 2016

Levy walk of population will generate new solution around the best solution. Population vector is modified using levy flight equation $x_{a b}^{t+1}$ i.e, belongs to $a^{\text {th }}$ nest and $b^{\text {th }}$ control variable. Here old value $x_{a b}$ is updated with respect to $f^{\text {th }}$ neighborhood's nest, using Eqn. (2.20) is used to select host nest position and the egg laid by cuckoo is evaluated.

\section{(c) Crossover}

Recently an efficient operator crossover has been designed for searching process [10].

$$
x_{a b}^{n e w}=(1-\lambda) \times x_{1 b}^{r e f}+\lambda \times x_{a b}^{\text {old }}
$$

Where $\lambda$ is the random number between $[0,1]$

Modified value $x_{a b}$ is obtained by crossover of old value and its reference value. After crossover check the control variable limits for all the population. If upper limit is violated set to the maximum value, lower limit is violated set to the minimum value and if it is within the limit keep as such.

\section{(d) Selection}

For this work sorting and ranking process is used. By comparing initial generation function vector and new function vector after performing crossover operator. Now modified function vector is obtained for new population, the minimum function value will be memorized. Now the function vectors sort by ascending order in which function values are ranked from minimum to maximum value. Then first rank function value and its corresponding population value are treated as best, and best population vector is given to the next generation.

\section{(e) Stopping criteria}

Whenever the number of current generations equals to the maximum number of generations specified then final solution is obtained.

\section{H. Load flow in the presence of capacitor}

Step 1: Read the line and load data for a distribution system

Step 2: Form the NIBC matrix from the node identification algorithm $[7,8]$

Step 3: Perform the load flows to find the system node voltages, line flows and total active and reactive power losses.

Step 4: Using the single objectives mentioned section II, i.e maximization of savings, minimization of voltage deviation, minimization of section current index, subjected to the constraints; perform the MCSA to achieve the objectives mentioned section II.

Step 5: Check the equality and inequality constraints as in section II-C.

Step 6: Print the size of capacitors and Objective function values.

Step 7: Stop

\section{Parameters of Proposed MCSA}

\begin{tabular}{clc}
\hline Proposed & Number of host nest & $\mathbf{5 0}$ \\
\cline { 2 - 3 } MCSA & Recombination constant & $\operatorname{rand}(0,1)$ \\
& Number of Iteration & 100 \\
& Levy flight constant $(\lambda)$ & $1 \leq \lambda \leq 3$ \\
& Levy flight constant $(\alpha)$ & $\operatorname{rand}(-1,1)$ \\
& Cross over constant $\left(\lambda_{\text {cross }}\right)$ & $\operatorname{rand}(0,1)$ \\
\hline
\end{tabular}

\section{J. Results and analysis}

To validate the developed methodology, a standard 15node radial distribution system has been chosen.

At first, load flow problem is solved. Later, to identify the effect of capacitors on system performance, the optimal locations to install capacitors are identified using power loss index (PLI) analysis. For this, PLI values are evaluated at each of the node using the procedure given in [11] and are arranged in descending order. Then, one capacitor is installed in highest PLI valued location and the optimal capacitor settings and total power losses are evaluated using the optimization procedure given in [11]. This process is repeated for two, three capacitor locations and the total power losses are evaluated. Finally, the number of locations which yields lowest power losses are considered as the optimum number of capacitor locations as in Table 1. Next, by installing capacitors in these optimum locations, the savings, voltage deviation and section current index objectives are optimized in the presence of capacitor individually using MCSA

Table.1 Optimum capacitor locations of 15-node RDS

\begin{tabular}{|c|c|c|}
\hline S. No & Locations & TPL value, $\mathbf{k W}$ \\
\hline 1 & 15 & 38.88012 \\
\hline $\mathbf{2}$ & $\mathbf{1 5 , 1 1}$ & $\mathbf{3 0 . 9 5 9 2 1}$ \\
\hline 3 & $15,11,4$ & 59.11021 \\
\hline
\end{tabular}

The detailed summary of the test results for capacitor placement are tabulated in Table.2.2. From this table, it is observed that, $28.1337 \mathrm{~kW}$ losses are reduced with capacitors when compared to without capacitors.

Table.2 Summary of test results for capacitor placement of 15-node RDS

\begin{tabular}{|c|c|c|}
\hline \multicolumn{2}{|c|}{ Description } & $\begin{array}{c}\text { With } \\
\text { Capacitor }\end{array}$ \\
\hline \multicolumn{2}{|c|}{ Capacitor locations } & 15,11 \\
\hline \multicolumn{2}{|c|}{ Capacitor size, kVAr } & $\begin{array}{c}103 \mathrm{kVAr} \\
930 \mathrm{kVAr}\end{array}$ \\
\hline \multirow{2}{*}{ TPL, kW } & Without & 59.10951 \\
\cline { 2 - 3 } & With & 30.97582 \\
\hline \multicolumn{2}{|c|}{ Loss reduction, kW } & 28.13373 \\
\hline
\end{tabular}

The single objective optimized results with savings, voltage deviation (Vdev) and section current index (SCI) as objectives for with and without capacitors using the developed MCSA is tabulated in Table.5, Table.6, Table.7. From these tables, it is identified that, with capacitor maximum benefit in terms of savings, $\mathrm{V}_{\mathrm{dev}}$ and $\mathrm{SCI}$ values is obtained when compared to without capacitor.

From Table.5 it is clear that, maximization of savings, increases the savings due to annual energy loss and there by the reserve capacity for demand growth which in turn increases the SCI value. It is also observed that, because of 
INTERNATIONAL JOURNAL OF INNOVATIVE RESEARCH IN ELECTRICAL, ELECTRONICS, INSTRUMENTATION AND CONTROL ENGINEERING

Vol. 4, Issue 2, February 2016

moderate capacitor compensation, moderate variation is observed in cost of capacitors, voltage deviation, total power losses, benefit due to released feeder capacity and benefit due to reduced demand, as compared to the other objectives of voltage deviation and section current index as in Table. 6 and Table.7.

From Table. 6 it observed that, minimization of voltage deviation increases to capacitors compensation. Hence, size of capacitors is increased more when compared to other objectives. This in turn increases the cost of capacitors installation. It is also observed that, due to minimization of voltage deviation, the total power losses are reduced which increases the benefit due to released feeder capacity and benefit due to reduced demand. In this case, moderate variation is observed for section current index and benefit due to losses because of the increased compensation, as compared to the other objectives of savings and section current index as in Table.5 and Table.7.

From Table.7 it is observed that, minimization of SCI value, the compensation required is decreased which in turn decreases the cost of capacitors and increases the total power losses and voltage deviations. Because of this, the system/feeder performance in terms of savings is decreased. It is also observed that, benefit due to released feeder capacity and reduced demand is decreased, as the capacitors compensation is very less, as compared to the other objectives of voltage deviation and savings as in Table.5 and Table.6.

Table.5 Single objective optimized results with capacitors of 15-node RDS

\begin{tabular}{|c|c|c|}
\hline \multirow{2}{*}{$\begin{array}{c}\text { Control } \\
\text { Parameters }\end{array}$} & \multicolumn{2}{|c|}{ With capacitors- Objective-Savings } \\
\hline & $\begin{array}{c}\text { Existing } \\
{[7]} \\
\end{array}$ & $\begin{array}{c}\text { Proposed } \\
\text { Maximization }\end{array}$ \\
\hline $\begin{array}{l}\mathrm{Q}_{\mathrm{C} 15} \\
\mathrm{kVAr}\end{array}$ & $\begin{array}{c}450(3), 450(6), \\
150(13), 150(15) \\
\end{array}$ & 450 \\
\hline $\mathrm{Q}_{\mathrm{C} 11}$, & ----- & 450 \\
\hline KP, Rs & $3,15,900$ & $3,01,963$ \\
\hline KF, Rs & ------ & 42,558 \\
\hline KE, Rs & |----- & 2,264 \\
\hline $\mathrm{KC}, \mathrm{Rs}$ & 22,680 & 40,986 \\
\hline Savings, & $2,93,220$ & $3,03,762$ \\
\hline Vdev, p.u. & ------ & 0.54598 \\
\hline SCI value & $\begin{array}{l}----- \\
-4\end{array}$ & 0.56341 \\
\hline TPL, kW & 30.4463 & 30.3164 \\
\hline
\end{tabular}

Table.6 Single objective optimized results with capacitors of 15-node RDS

\begin{tabular}{|c|c|c|}
\hline \multirow{2}{*}{$\begin{array}{c}\text { Control } \\
\text { Parameters }\end{array}$} & \multirow{2}{*}{$\begin{array}{c}\text { Without } \\
\text { capacitor }\end{array}$} & $\begin{array}{c}\text { Woltage deviation } \\
\text { (Vdev) (p.u.) } \\
\text { Minimization }\end{array}$ \\
\hline $\mathrm{Q}_{\mathrm{C} 15}, \mathrm{kVAr}$ & - & 750 \\
\hline $\mathrm{Q}_{\mathrm{C} 11}, \mathrm{kVAr}$ & - & 450 \\
\hline $\mathrm{KP}, \mathrm{Rs}$ & $1,75,829$ & $2,85,776$ \\
\hline $\mathrm{KF}, \mathrm{Rs}$ & 24,714 & 46,538 \\
\hline $\mathrm{KE}, \mathrm{Rs}$ & 1,318 & 2,143 \\
\hline KC, Rs & 0 & 54,648 \\
\hline Savings, Rs & $1,78,432$ & $2,77,880$ \\
\hline Vdev, p.u. & 0.8784 & 0.3706 \\
\hline SCI value & 0.5752 & 0.3927 \\
\hline TPL, $\mathrm{kW}$ & 59.5954 & 26.8253 \\
\hline
\end{tabular}

Table.7 Single objective optimized results with capacitors of 15-node RDS

\begin{tabular}{|c|c|c|}
\hline Control & Without & With capacitors \\
\cline { 3 - 3 } Parameters & capacitor & Section current \\
\hline $\mathrm{Q}_{\mathrm{C} 15}, \mathrm{kVAr}$ & - & 250 \\
\hline $\mathrm{Q}_{\mathrm{C} 11}, \mathrm{kVAr}$ & - & 250 \\
\hline $\mathrm{KP}, \mathrm{Rs}$ & $1,75,829$ & $2,46,312$ \\
\hline $\mathrm{KF}, \mathrm{Rs}$ & 24,714 & 19,447 \\
\hline KE, Rs & 1,318 & 1,847 \\
\hline KC, Rs & 0 & 13,662 \\
\hline Savings, Rs & $1,78,432$ & $2,52,283$ \\
\hline Vdev, p.u. & 0.8784 & 0.8151 \\
\hline SCI value & 0.5752 & 0.1673 \\
\hline TPL, kW & 59.11 & 55.38 \\
\hline
\end{tabular}

\section{IV.CONCLUSION}

A modified Cuckoo search Algorithm is used for optimal placement of capacitor with three objective functions taking into consideration of the economic and the technical factors of the system into consideration independently, as well as satisfying the constraints. The authors were able to obtain maximization of net savings for the system, minimization of voltage deviation and minimization of section current index for capacitors placement in a standard 15-node radial distribution system. To validate the improvement in optimal placement of capacitor by MCSA, the results have been compared with the Existing method. The results reveal that a better Total active power loss and the net savings were obtained than the existing method.

\section{REFERENCES}

[1] José Federico Vizcaino González, Christiano Lyra, Fábio Luiz Usberti, “A pseudo-polynomial algorithm for optimal capacitor placement on electric power distribution networks" European Journal of Operational Research (Elsevier), Volume 222, Issue 1, Pages 149-156, 1 October 2012.

[2] Xin-mei Yu, Xin-yin Xiong and Yao-wu Wu, "A PSO-based ap-proach to optimal capacitor placement with harmonic distortion consideration" Electric Power Systems Research (Elsevier), Volume 71, Issue 1, Pages 27-33, September 2004.

[3] A. Kalyuzhny, G. Levitin, D. Elmakis and H. Ben-Haim, "System approach to shunt capacitor allocation in radial distribution systems" Electric Power Systems Research (Elsevier), Volume 56, Issue 1, Pages 51-60, 5 October 2000.

[4] Ji-Pyng Chiou, Chung-Fu Chang and Ching-Tzong Su, "Capaci-tor placement in large-scale distribution systems using variable scaling hybrid differential evolution" International Journal of Electrical Power \& Energy Systems (Elsevier), Volume 28, Issue 10, Pages 739-745, December 2006

[5] R. Srinivasas Rao, S.V.L. Narasimham, M. Ramalingaraju, "Optimal capacitor placement in a radial distribution system using Plant Growth Simulation Algorithm" International Journal of Electrical Power \& Energy Systems (Elsevier), Volume 33, Issue 5, Pages 1133-1139, June 2011.

6] S.M. Tabatabaei and B. Vahidi, "Bacterial foraging solution based fuzzy logic decision for optimal capacitor allocation in radial distribution system" Electric Power Systems Research (Elsevier), Volume 81, Issue 4, Pages 1045-1050, April 2011.

[7] Das D, Kothari D P, Kalam A. Simple and efficient method for load flow solution of radial distribution networks. Int J Electr Power Energy Syst 1995; 17(5): 335-46.

[8] Teng Jen-Hao. A direct approach for distribution system load flow solutions, IEEE Trans Power Deliver 2003;18(3):882-7.

[9] X.S. Yang, S. Deb, Cuckoo search via Levy flights, World Congress on Nature \& Biologically Inspired Computing (Nabic 2009), December 2009, India, IEEE Publications, USA, pp. 210-214, 2009

[10] X.S. Yang, S. Deb, Engineering optimization by cuckoo search, Int. J. Mathematical Modelling and Numerical Optimization, Vol. 1, No. 4, pp.330-343 2010.

[11] Reddy V.V.K, Sydulu.M, "Index and GA based Optimal location and Sizing of distribution system Capacitors", IEEE Power Eng. Soc. General Meet. June 2007.

[12] Das D, Kothari D P, Kalam A. Simple and efficient method for load flow solution of radial distribution networks. Int J Electr Power Energy Syst, Vol.17, No.5, pp.335-46, 1995.

[13] Teng Jen-Hao. A direct approach for distribution system load flow solutions, IEEE Trans Power Deliver Vol.18, No.3, pp.882-7, 2003. 